\title{
Valorización Agrícola de Purines Porcinos Procesados con Aserrín de Pino
}

María T. Varnero, Susana Muñoz y Roberto Zúñiga

Universidad de Chile, Facultad de Ciencias Agronómicas, Casilla 1004, Santiago-Chile

(e-mail: mvarnero@uchile.cl, smunoz@uchile.cl, rzunigas@vtr.net)

\section{Resumen}

Con el objeto de regular la humedad excesiva en purines porcinos y obtener un material estabilizado, se implementó una metodología de tratamiento utilizando aserrín de pino como estructurante, en dosis de $1.0,1.5$ y $2.0 \mathrm{~m}^{3}$. Los purines crudos se distribuyeron sobre estas unidades, manteniéndolos cinco días en reposo. Con este material, se formaron pilas en condiciones aeróbicas, realizándose volteos periódicos. Al término del ensayo, se observó en todos los tratamientos una disminución de la salinidad. Los altos contenidos de materia orgánica y de la relación $\mathrm{C} / \mathrm{N}$ observados en estas mezclas, sumado a la disminución de la humedad del material estructurante, permite suponer que se podría reutilizar con nuevas cargas de purines de cerdo. El producto final tiene características adecuadas en términos de relación amonio/nitrato, para establecer el desarrollo de cultivos sin restricciones y los niveles de coliformes fecales disminuyen drásticamente.

\section{Agricultural Valorization of Swine Manure Processed with Pine Sawdust}

\begin{abstract}
In order to control excessive humidity of swine manure and to obtain a stabilized material, pine sawdust was used as bulk material. Several doses of pine sawdust were evaluated: 1.0, 1.5 and 2.0 $\mathrm{m}^{3}$. Raw manure was spread and maintained in rest for five days. Piles of this material were constructed under aerobic conditions and periodically turned. At the end of the assay all treatments showed a decrease of the salinity. The high contents of organic matter and the $\mathrm{C} / \mathrm{N}$ ratio of these mixtures, and the decrease of the humidity of the bulk material allows assuming that this materia could be reused with new charges of swine manure. The end product obtained has appropriate characteristics in terms of ammonium/nitrate ratio for establishing cultures without restrictions and the fecal coliform levels decrease drastically.
\end{abstract}

Keywords: manure processing, organic conditioning, clean production, stabilized manure 


\section{INTRODUCCIÓN}

La evolución en los últimos años de las explotaciones ganaderas intensivas, fundamentalmente de cerdos, esta orientada hacia unidades con un gran número de animales (ODEPA, 2006), sin terreno que permita absorber la cantidad de residuos que se genera, lo que está planteando serios problemas en el manejo de los mismos. Los purines de cerdo presentan características que determinan que sean grandes contaminantes, afectando al medio ambiente y al ser humano, producto de un manejo y tratamiento inadecuado. En Chile no existen normativas específicas para tratar estos purines; no obstante, si las empresas generan un mal uso, pueden llegar a infringir algunas normas de la legislación chilena que afectan a la agricultura, la salud y obras públicas, entre otras. El tratamiento de purines porcinos es un campo relativamente nuevo dentro de los tratamientos de residuos (Peralta et al., 2005) y aún no existe una tecnología completamente consolidada que sirva como referencia para tratar estos residuos. El método más conocido es su uso como fertilizante incorporado al suelo (Faz et al., 2003; Miralles et al., 2007), para aprovechar el contenido de nutrientes y de materia orgánica que poseen, y disminuir los costos de fertilización de cultivos. Los principales elementos que definen los purines son: materia orgánica $(\mathrm{MO})$, nitrógeno $(\mathrm{N})$, fósforo $(\mathrm{P})$, potasio $(\mathrm{K})$, calcio $(\mathrm{Ca})$, magnesio $(\mathrm{Mg})$ sodio $(\mathrm{Na})$, cobre $(\mathrm{Cu})$, cobalto $(\mathrm{Co})$; boro $(\mathrm{B})$ fluor $(\mathrm{F})$, molidebno (Mo) entre otros. Sin embargo, uno de los mayores problemas radica en su elevado contenido de agua (Farres, 2006), lo que encarece su gestión; transporte, aplicación y tratamiento. Es así como planteles eficientes en el uso del agua, poseen relaciones excretas/ agua de lavado no mayores a $1 / 4$ (4 litros de agua de lavado por 1 litro de excreta) y planteles muy rústicos llegan a tener relaciones de 1/18 lo que obviamente cambia la planificación en el manejo de los purines (Peralta et al., 2005). Otro inconveniente que presenta la aplicación de purines en el suelo es el impacto ambiental, debido a los riesgos que se corren respecto de la eutrofización, la contaminación con patógenos, los malos olores y la proliferación de insectos, ya que si la materia orgánica llega a las masas de agua superficiales, o freáticas por escorrentía, por vertido inadecuado, el efecto inmediato es una pérdida de oxígeno disuelto y el inicio del proceso de eutrofización con la consiguiente contaminación de las mismas.

Actualmente se ha renovado el interés por el procesamiento de purines en condiciones anaeróbicas, con la finalidad de generar biogás junto con un material digerido y estabilizado, para incorporar al suelo como bioabono (Kirkbride, 2007). En Estados Unidos, se han realizado estudios sobre la utilización de los purines de cerdo mezclados con material de origen vegetal para su posterior compostaje. Los resultados preliminares muestran la obtención de un compost de buena calidad, con una relación $\mathrm{C} / \mathrm{N}$ de alrededor de 12 y con un 48\% de materia seca (Boyette et al., 2005). Estas mezclas buscan establecer un buen balance entre el carbono y el nitrógeno y la regulación de la humedad que generalmente es excesiva en purines y sus derivados. Sin embargo, también se han implementado ensayos, separando la fracción líquida mediante polímeros, para compostar posteriormente la fracción sólida mezclada con desechos urbanos (Smicikles et al., 2008). El efluente que se obtiene luego de la separación sólido - líquido, se utiliza generalmente en lagunas de fermentación anaeróbica, ya que éste aun conserva una cantidad importante de nutrientes que pueden ser utilizados por otros microorganismos (Ramírez, 2006). El compost obtenido puede ser utilizado como enmienda para el suelo, fertilizante orgánico o como parte de un sustrato usado en la producción de plantas en vivero (Vanotti, 2005).

En este trabajo se propuso la implementación de una planta piloto de tratamiento de purines de cerdo, usando como material estructurante aserrín, cuyo objetivo principal fue procesar y obtener un material estabilizado, libre de patógenos y malos olores; con la finalidad de utilizarlo como materia prima en la elaboración de compost u otro uso que se considere apropiado.

\section{MATERIALES Y MÉTODOS}

El lugar donde se realizó este ensayo, fue en un predio agrícola, ubicado en la zona de San Vicente de Naltahua, comuna de Isla de Maipo, Región Metropolitana (Chile), que posee 500 madres y un total aproximado de 11.000 animales; utilizando como materia prima el purín completo o crudo sin ningún tipo de procesamiento previo, y como material estructurante, aserrín de pino. Se realizó una 
caracterización física, química y microbiológica a los materiales (TMECC, 2002). Las unidades de tratamiento o contenedores se construyeron con madera aglomerada dimensionada y se forraron por dentro con nylon de alta resistencia para evitar la fuga de material liquido. Las dimensiones de estas unidades fueron: 2,5 m de largo x 0,6 $\mathrm{m}$ de alto y 1,5 $\mathrm{m}$ de ancho; correspondiente a un volumen de $2,25 \mathrm{~m}^{3}$ por unidad de tratamiento. Adicionalmente se implementó una cancha de secado, de $100 \mathrm{~m}^{2}$, donde se utilizó nylon de alta resistencia a modo de piso para recibir el material durante el proceso de secado. Ensayos de laboratorio previos, demostraron que en términos de volumen, el $\mathrm{m}^{3} \mathrm{de}$ aserrín es capaz de retener $1.5 \mathrm{~m}^{3}$ de purín. Esta relación se consideró para generar el protocolo de operación propuesto.

El diseño experimental utilizado fue un modelo de cuadrado latino con 3 tratamientos y 3 repeticiones de cada uno. Los purines se distribuyeron sobre las unidades de tratamiento, las que corresponden a dosis de 1,$0 ; 1,5$ y $2,0 \mathrm{~m}^{3}$ de aserrín para el tratamiento $1 ; 2$ y 3 respectivamente. Todas las unidades se cargaron diariamente con aserrín en una cantidad de $0.5 \mathrm{~m}^{3}$, y con purín en una cantidad de $0.5 \mathrm{~m}^{3}$, hasta completar la cantidad de aserrín destinada para cada tratamiento, totalizando 2 días para el tratamiento 1, hasta 4 días para el tratamiento 3 . Esto se realizó diariamente con el fin de encontrar la saturación de humedad máxima del aserrín. Después de 5 días de reposo en las unidades de tratamiento, se dispuso el material, en la cancha de secado, donde se formaron pilas distribuidas según el diseño experimental propuesto, comenzando el proceso de descomposición de las materias primas, junto con el secado paulatino de las mezclas. Se realizaron volteos del material en función de la temperatura interna de cada pila; además de controles periódicos de humedad, $\mathrm{pH}$, relación amonio/nitrato, contenido de sales, medido como conductividad eléctrica (C.E.) y de materia orgánica (MO), de cada tratamiento (TMECC, 2002). Al término del ensayo, se realizó un análisis físico, químico y biológico al producto obtenido. Se realizó análisis de varianza (ANDEVA) para determinar si existe diferencia entre los tratamientos en variables individuales como, contenido de materia orgánica (MO), contenido de materia seca (MS), relación carbono/nitrógeno $(\mathrm{C} / \mathrm{N})$ y relación amonio/nitrato $\left(\mathrm{N}-\mathrm{NH}_{4}{ }^{+} / \mathrm{N}-\mathrm{NO}_{3}{ }^{-}\right)$.

\section{RESULTADOS Y DISCUSIÓN}

La caracterización previa que se realizó a los purines frescos indicó que los altos niveles de coliformes fecales encontrados en el purín $\left(8^{*} 10^{7}\right)$, representan un riesgo sanitario importante. Distintos muestreos realizados en criaderos chilenos indican que en general los valores de coliformes fecales medidos como NMP/g fluctúan entre $1.6 \times 10^{7}$ y $2.3 \times 10^{8}$ (Tapia, 2006). En cambio, los análisis referidos a metales pesados, como por ejemplo, arsénico $(<0,01 \mathrm{mg} / \mathrm{L})$ y mercurio $(<0,001)$ no exceden los niveles aceptados por las normas internacionales (USEPA, 1995).

En la determinación de los elementos esenciales (macronutrientes y oligoelementos) se aprecian valores de interés agronómico, constituyendo un aporte si se incorporaran al suelo. Sin embargo, el nitrógeno es uno de los macronutrientes de mayor importancia, debido a la dinámica que se establece en los tratamientos aeróbicos realizados, por lo tanto, en este estudio se realizó un seguimiento de sus fracciones. El contenido de nitrógeno amoniacal $\left(\mathrm{N}^{-} \mathrm{NH}_{4}{ }^{+}\right)$igual a $447 \mathrm{mg} / \mathrm{L}$, constituye cerca del $50 \%$ del nitrógeno total $\left(\mathrm{N}_{\mathrm{T}}\right)$ determinado (Tabla 1), el cual representa la suma de la fracción orgánica y mineral. En esta fracción mineral, el contenido de nitrógeno nítrico $\left(\mathrm{N}-\mathrm{NO}_{3}{ }^{-}\right)$, no supera el $3 \%$; con lo cual se obtiene una relación de amonio/nitrato inadecuada y por lo tanto, también afectaría negativamente el desarrollo de cultivos, debido a la toxicidad que produce el amonio en concentraciones altas en los tejidos vegetales. Esto, se corrobora con los valores de conductividad eléctrica obtenidos (Tabla 1) que indican una elevada salinidad, lo que constituye una limitante para la utilización directa de purines en la producción agrícola, a pesar que este valor se considera dentro del promedio observado en diferentes planteles en el país (Tapia, 2006). Por otra parte, la relación $\mathrm{C} / \mathrm{N}$ y el contenido de sólidos totales (ST) presente en los purines (Tabla 1), constituyen un factor limitante para el manejo y uso de estos residuos.

Tabla 1; Caracterización química de purines porcinos frescos.

\begin{tabular}{|c|c|c|c|c|c|c|c|c|c|}
\hline $\mathrm{pH}$ & $\begin{array}{c}\mathrm{C} . \mathrm{E} . \\
(\mathrm{dS} / \mathrm{m})\end{array}$ & $\begin{array}{c}\mathrm{ST} \\
(\%)\end{array}$ & $\begin{array}{c}\mathrm{MO} \\
(\mathrm{g} / \mathrm{kg})\end{array}$ & $\begin{array}{c}\text { C orgánico } \\
(\mathrm{g} / \mathrm{kg})\end{array}$ & $\begin{array}{c}\mathrm{N}_{\mathrm{T}} \\
(\mathrm{g} / \mathrm{kg})\end{array}$ & $\mathrm{C} / \mathrm{N}$ & $\begin{array}{c}\mathrm{N}^{-\mathrm{NH}_{4}}+ \\
(\mathrm{mg} / \mathrm{L})\end{array}$ & $\begin{array}{c}\mathrm{N}^{-\mathrm{NO}_{3}} \\
(\mathrm{mg} / \mathrm{L})\end{array}$ & $\begin{array}{c}\mathrm{N}^{-\mathrm{NH}_{4}}{ }^{+} / \mathrm{N}- \\
\mathrm{NO}_{3}^{-}\end{array}$ \\
\hline 6,6 & 6,2 & 2,0 & 7,1 & 3,9 & 0,9 & 4,3 & 447,0 & 14,0 & 32 \\
\hline
\end{tabular}


Esta situación requiere de la adición de residuos leñosos, como el aserrín (Tabla 2) para equilibrar esta relación $\mathrm{C} / \mathrm{N}$, junto con absorber el elevado contenido de agua que tienen los purines.

Tabla 2: Caracterización química del aserrín de pino.

\begin{tabular}{|c|c|c|c|c|c|c|}
\hline $\mathrm{pH}$ & $\begin{array}{c}\text { C.E. } \\
(\mathrm{dS} / \mathrm{m})\end{array}$ & $\begin{array}{c}\mathrm{MS} \\
(\%)\end{array}$ & $\begin{array}{c}\text { MO } \\
(\mathrm{g} / \mathrm{kg})\end{array}$ & $\begin{array}{c}\text { C orgánico } \\
(\mathrm{g} / \mathrm{kg})\end{array}$ & $\begin{array}{c}\mathrm{N}_{\mathrm{T}} \\
(\mathrm{g} / \mathrm{kg})\end{array}$ & $\mathrm{C} / \mathrm{N}$ \\
\hline 5,3 & 0,18 & 58 & 995 & 553 & 1.5 & 369 \\
\hline
\end{tabular}

Se puede observar que al mezclar y bioprocesar estos materiales (Tabla 3), se incrementan los valores de la relación $\mathrm{C} / \mathrm{N}$ dentro de rangos no adecuados para optimizar la actividad microbiana de estos sistemas. Sin embargo, los altos índices de salinidad presentes en el purín, así como los elevados valores de la relación amonio/nitrato (Tabla 1), disminuyeron considerablemente en todos los tratamientos.

Tabla 3: Caracterización química de materiales bioprocesados al inicio ensayo.

\begin{tabular}{|c|c|c|c|c|c|c|c|c|c|c|}
\hline Tratamientos & $\mathrm{pH}$ & $\begin{array}{c}\mathrm{C} . \mathrm{E} . \\
(\mathrm{dS} / \mathrm{m})\end{array}$ & $\begin{array}{c}\mathrm{MS} \\
(\%)\end{array}$ & $\begin{array}{c}\mathrm{MO} \\
(\mathrm{g} / \mathrm{kg})\end{array}$ & $\begin{array}{c}\mathrm{C} \text { orgánico } \\
(\mathrm{g} / \mathrm{kg})\end{array}$ & $\begin{array}{c}\mathrm{N}_{\mathrm{T}} \\
(\mathrm{g} / \mathrm{kg})\end{array}$ & $\mathrm{C} / \mathrm{N}$ & $\begin{array}{c}\mathrm{N}_{-}^{-} \\
\mathrm{NH}_{4}^{+} \\
(\mathrm{mg} / \mathrm{L})\end{array}$ & $\begin{array}{c}\mathrm{N}^{+} \mathrm{NO}_{3}{ }^{-} \\
(\mathrm{mg} / \mathrm{L})\end{array}$ & $\begin{array}{c}\mathrm{N}^{-\mathrm{NH}_{4}}{ }^{+} \mathrm{I} \\
\mathrm{N}^{-\mathrm{NO}_{3}}{ }^{-}\end{array}$ \\
\hline $\mathrm{T} 1$ & 5,4 & 0,9 & 23 & 956 & 531 & 4,0 & 133 & 770 & 180 & 4,3 \\
\hline T2 & 5,5 & 0,9 & 28 & 969 & 538 & 3,7 & 145 & 550 & 42 & 13,1 \\
\hline T3 & 5,5 & 1,0 & 25 & 963 & 535 & 4,1 & 131 & 679 & 156 & 4,4 \\
\hline
\end{tabular}

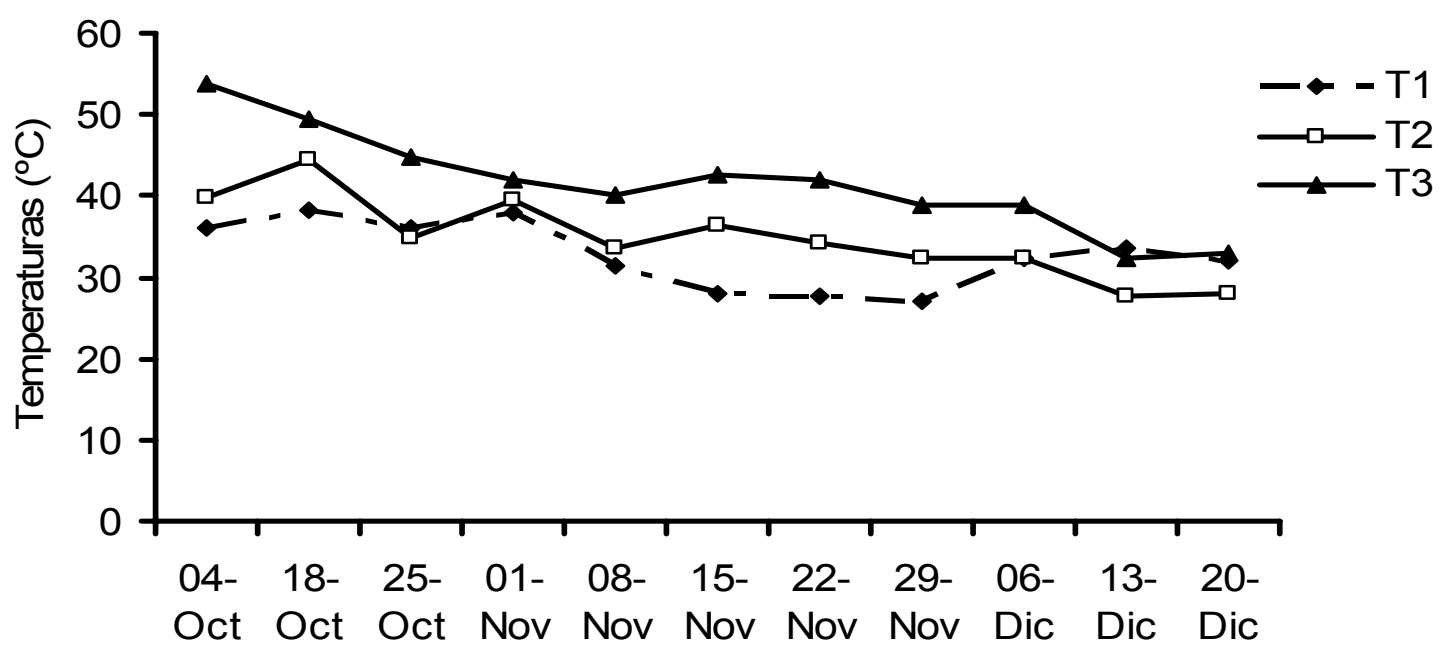

Fig. 1: Evolución de la temperatura durante el bioprocesamiento de las mezclas purines-aserrín.

En la Figura 1 se pueden observar las fases térmicas obtenidas durante el ensayo. Una primera etapa mesofilica, aproximadamente de una semana, luego una etapa termofílica donde las temperaturas se elevaron en los tres tratamientos a sus respectivos máximos térmicos. El primero en alcanzar este máximo fue el tratamiento 3, lo que se relaciona con el mayor tamaño de la pila o capacidad de aislamiento térmico según Mathur (1991), lo que impide la perdida de calor desde el interior de la misma. Los tratamientos 1 y 2 alcanzaron sus pick más tarde que el tratamiento 3 y se mantuvieron en temperaturas termófilas por un tiempo más reducido. Esto se podría atribuir al menor tamaño de las pilas de estos tratamientos en comparación con el tratamiento 3 , y además, porque presentaban una relación $\mathrm{C} / \mathrm{N}$ levemente superior que la obtenida en el tratamiento 3 (Tabla 3), lo que se traduciría en una menor producción de calor por la falta de nitrógeno para el crecimiento y desarrollo de los microorganismos.

En general luego de los volteos, la temperatura se incrementó debido a la entrada de oxigeno al sistema, lo que generó un aumento en la actividad microbiana para utilizar los sustratos susceptibles 
a la degradación, hasta que éstos se agotaron y ya no hubo respuesta al volteo. Las temperaturas descendieron gradualmente llegando finalmente a temperaturas muy similares a las del ambiente. Esto se produce básicamente por el agotamiento de los sustratos lábiles, lo que conlleva a una baja tasa de actividad microbiana.

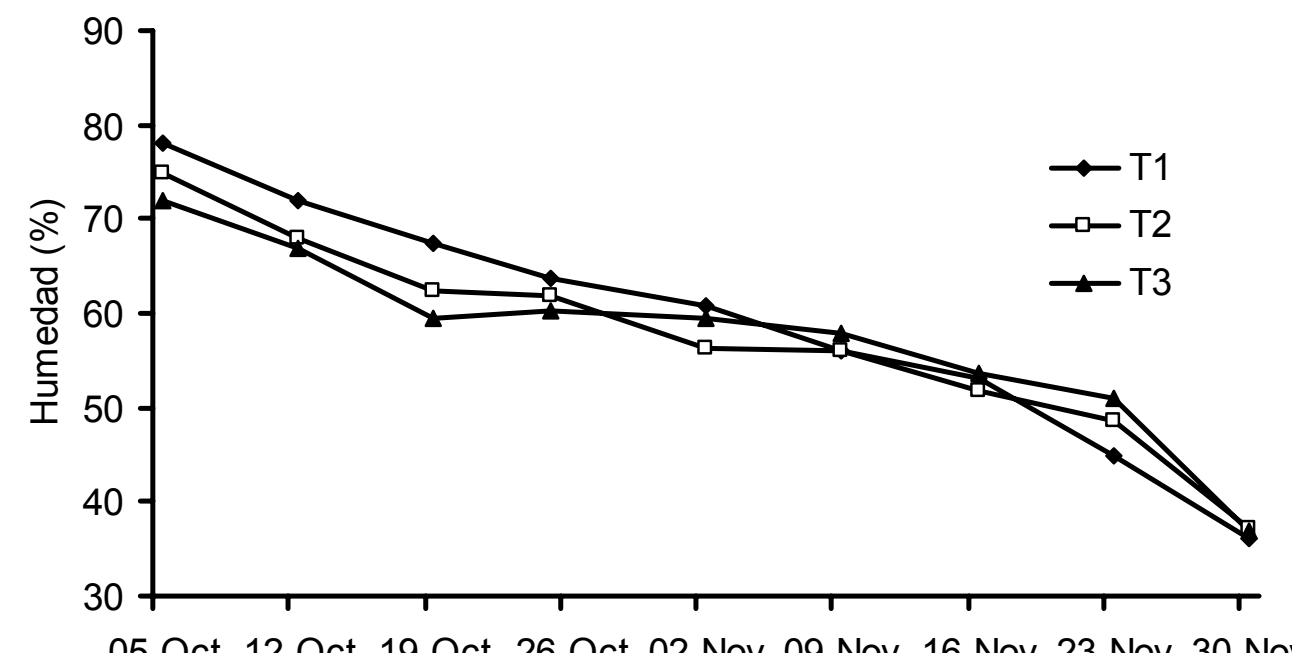

Fig. 2: Contenido de humedad del material en los tres tratamientos durante el bioprocesamiento.

En la Figura 2 se observa que todos los tratamientos comenzaron con porcentajes de humedad entre 70 y $80 \%$ lo que influyó negativamente en el proceso de degradación, esto se explica por la disminución de los espacios usados para el intercambio gaseoso lo que produce condiciones anaeróbicas y a la vez malos olores. Esta situación se expresó en las pilas, en forma de coloración anaranjada sobre todo en los centros, donde este color se acentuó y se logró percibir olores muy distintos al olor preponderante en el resto de la pila. Luego de 2 semanas de iniciado el bioproceso, la humedad se situó en los rangos que se consideran adecuados para una buena degradación de la materia orgánica (50-65\%). Esto, a su vez, permitió el inicio de la etapa termofílica y aceleró el secado del material debido al aumento de las temperaturas y las pérdidas de humedad que se generan luego de los volteos. Junto con lo anterior, se observó en todas las pilas, la disminución de insectos vectores de enfermedades como mosquitos y zancudos, llegando a desaparecer por completo antes del primer mes de iniciado el ensayo.

La humedad descendió gradualmente y se situó entre un 35 a 50\% al final del bioproceso para todos los tratamientos, con lo cual se puso fin al mismo. Los tratamientos 2 y 3 presentaron un mayor contenido de materia seca, lo que se puede atribuir a una mejor capacidad aislante, inducida por el tamaño de las pilas, lo que produce una menor perdida de calor desde el interior de éstas. Sin embargo, no existen diferencias significativas entre tratamientos. Las mayores diferencias entre el material bioprocesado inicial y final, se observan en todos los tratamientos en el contenido de amonio, de nitrato y materia seca (Tablas 3 y 4). Tanto la $\mathrm{MO}$ como el $\mathrm{C}$ orgánico no sufrieron grandes variaciones, lo cual indica la presencia de un alto contenido de material orgánico celulósico y hemicelulósico de difícil degradación.

Tabla 4: Caracterización química de los materiales bioprocesados al término del ensayo. Letras minúsculas iguales indican que no existen diferencias significativas entre los tratamientos, con un nivel de significancia del $5 \%$.

\begin{tabular}{|c|c|c|c|c|c|c|c|c|c|c|}
\hline Tratamientos & $\mathrm{pH}$ & $\begin{array}{c}\text { C.E. } \\
(\mathrm{dS} / \mathrm{m})\end{array}$ & $\begin{array}{c}\mathrm{MS} \\
(\%)\end{array}$ & $\begin{array}{c}\mathrm{MO} \\
(\mathrm{g} / \mathrm{kg})\end{array}$ & $\begin{array}{c}\mathrm{C} \text { orgánico } \\
(\mathrm{g} / \mathrm{kg})\end{array}$ & $\mathrm{N}_{\mathrm{T}}$ & $\mathrm{C} / \mathrm{N}$ & $\begin{array}{c}\mathrm{N}- \\
\mathrm{NH}_{4}^{+} \\
(\mathrm{mg} / \mathrm{L})\end{array}$ & $\begin{array}{c}\mathrm{N}^{+} \mathrm{NO}_{3}^{-} \\
\left(\mathrm{mg} / \mathrm{L}^{-}\right.\end{array}$ & $\begin{array}{c}\mathrm{N}- \\
\mathrm{NH}_{4}^{+} / \\
\mathrm{N}^{+} \mathrm{NO}_{3}{ }^{-}\end{array}$ \\
\hline $\mathrm{T} 1$ & $5,2 \mathrm{a}$ & $0,9 \mathrm{a}$ & $53 \mathrm{a}$ & $946 \mathrm{a})$ & $526 \mathrm{a}$ & $3,4 \mathrm{a}$ & $155 \mathrm{a}$ & $226 \mathrm{~b}$ & $103 \mathrm{a}$ & $2,2 \mathrm{a}$ \\
\hline $\mathrm{T} 2$ & $5,0 \mathrm{a}$ & $0,9 \mathrm{a}$ & $63 \mathrm{~b}$ & $942 \mathrm{a}$ & $523 \mathrm{a}$ & $3,5 \mathrm{a}$ & $150 \mathrm{a}$ & $161 \mathrm{a}$ & $128 \mathrm{~b}$ & $1,3 \mathrm{a}$ \\
\hline $\mathrm{T} 3$ & $5,0 \mathrm{a}$ & $1,0 \mathrm{a}$ & $64 \mathrm{~b}$ & $932 \mathrm{a}$ & $518 \mathrm{a}$ & $4,3 \mathrm{a}$ & $121 \mathrm{~b}$ & $189 \mathrm{a}$ & $140 \mathrm{~b}$ & $1,4 \mathrm{a}$ \\
\hline
\end{tabular}


Los tratamientos no presentaron diferencias estadísticas en sus contenidos de MO finales (Tabla 4); observándose que el contenido es elevado si se compara con los valores obtenidos en un compost elaborado usando la fracción sólida de purines de cerdo con paja de trigo, cuyos valores fluctuaron entre 50 y $55 \%$ de materia orgánica (Cortina et al., 2001). La relación $\mathrm{C} / \mathrm{N}$ registrada en el material final (Tabla 4), aumenta en los tratamientos 1 y 2 con relación a los valores que presentan las mezclas al inicio del ensayo (Tabla 3); en cambio, el tratamiento 3 disminuye la relación $\mathrm{C} / \mathrm{N}$ en comparación con el material inicial (Tabla 3) y es estadísticamente diferente de los otros dos tratamientos. Esto indicaría que se ha producido una mejor bioxidación de los materiales carbonados resistentes que posee el aserrín de pino, atribuible al mayor volumen que tienen las pilas del tratamiento 3. Sin embargo, según la NCh 2880 (INN, 2004), los valores de C/N indican que este material aún no se puede considerar maduro o estabilizado.

La relación amonio/nitrato disminuyó a niveles que están en el rango requerido para establecer la madurez química de materiales orgánicos tratados mediante compostaje (INN, 2004). Esto es importante ya que el nitrógeno amoniacal en exceso produce toxicidades en los tejidos vegetales. Sin embargo, según Zucconi y De Bertoldi (1987), si esta relación supera la unidad implica que aun existen materiales orgánicos no estabilizados. Para este análisis se contó solo con 3 datos por tratamiento lo que hace menos preciso el análisis estadístico, no observándose diferencias estadísticas entre los tratamientos; sin embargo, si se aprecia para el tratamiento 1 con relación al 2 y 3 , cuando se analiza los contenidos de amonio y nitrato por separado.

Tabla 5: Índice de germinación (IG) en semillas de rabanito (Raphanus sativum) y estabilidad biológica medido como desprendimiento de $\mathrm{CO}_{2}$ en los materiales bioprocesados.

\begin{tabular}{|c|c|c|}
\hline Tratamientos & $\begin{array}{c}\text { IG en semillas de rabanito } \\
(\%)\end{array}$ & $\begin{array}{c}\text { Desprendimiento de } \mathrm{CO}_{2} \\
\text { (mg C-CO } / \mathrm{g} \mathrm{M} . \mathrm{O} / \mathrm{día})\end{array}$ \\
\hline T1 & 100 & 0,18 \\
\hline T2 & 100 & 0,08 \\
\hline T3 & 98 & 0,22 \\
\hline
\end{tabular}

La Tabla 5 muestra que el porcentaje del índice de germinación (IG) obtenido para semillas de rabanito (Raphanus sativum) es de 100\% tanto para el tratamiento 1 como para el 2 y de $98 \%$ para el tratamiento 3. Esto implica que los tratamientos no presentan toxicidad para rabanito según la escala propuesta por Zucconi et al. (1981), ya que con índices de germinación mayores a 80\% se considera que el material no contiene sustancias toxicas o que ellas están en muy baja concentración. Se sabe que el rabanito es una especie sensible a la salinidad (Varnero et al., 2007), esto explica los altos índices de germinación obtenidos en los ensayos, ya que los valores de conductividad eléctrica obtenidos en todos los tratamientos disminuyeron, apreciándose un bajo nivel de salinidad (Tabla 4). De acuerdo a estos resultados y según la NCh 2880, el material bioprocesado se consideraría maduro (INN, 2004). Por otra parte, al determinar el grado de estabilidad de estos materiales (Tabla 4), mediante el desprendimiento de $\mathrm{CO}_{2}$ (Varnero et al., 2005), se observaron en los tres tratamientos valores inferiores a los exigidos para los materiales biológicos estables $(\leq 8 \mathrm{mg}$ de CO2/g de materia orgánica/día) según la NCh 2880 (INN, 2004). Esta baja actividad biológica se debe probablemente a que las fracciones orgánicas que persisten en el material bioprocesado son muy resistentes y por lo tanto poco biodegradables lo que no permite sustentar una población microbiana mesofílica activa (Varnero et al., 2007).

Los resultados obtenidos guardan relación con la naturaleza de la mezcla, ya que el aserrín por si solo presenta una marcada estabilidad biológica (Varnero et al., 2005). A la vez que, el purín aporta una gran cantidad de agua y poca materia orgánica la cual se degradada rápidamente

Tabla 6:. Conteo coliformes fecales de los materiales bioprocesados al término del ensayo.

\begin{tabular}{cccc}
\hline Tratamientos & Parámetro & Expresado como & Valor medido \\
\hline T1 & Coliformes fecales & $\mathrm{NMP} / \mathrm{g}$ & $17 \times 10^{4}$ \\
\hline T2 & Coliformes fecales & $\mathrm{NMP} / \mathrm{g}$ & $1.3 \times 10^{3}$ \\
\hline T3 & Coliformes fecales & $\mathrm{NMP} / \mathrm{g}$ & $0.2 \times 10^{3}$ \\
\hline
\end{tabular}


Los resultados presentados en la Tabla 6 con relación al número más probable por gramo (NPM/g) de coliformes fecales muestran diferencias notorias entre los tres tratamientos. Según la Nch 2880 (INN, 2004) el valor medido en el tratamiento 3 (200 NMP/g) permite calificar como aceptable este material en cuanto a su sanidad microbiológica. Los otros 2 tratamientos restantes poseen valores que se escapan del rango permitido por esta norma $(<1000 \mathrm{NMP} / \mathrm{g})$. Las diferencias se deben básicamente a las menores temperaturas registradas en estos tratamientos, ya que según explica Trautmann y Olyncin (2000), para obtener una reducción significativa de los patógenos durante el proceso de degradación es necesario alcanzar temperaturas del orden de los $55^{\circ} \mathrm{C}$ por lo menos durante 4 horas, condición que no se cumplió para estos tratamientos.

\section{CONCLUSIONES}

Los purines presentan propiedades químicas y biológicas que limitan su uso directo sobre el suelo, contaminando fuentes de agua subterránea y superficial. El aserrín presenta características deseables para su empleo como material estructurante en la mezcla con purín. Además permite la absorción de la elevada cantidad de agua que posee este residuo. El volumen de las pilas influye visiblemente en el proceso, siendo un parámetro fundamental para alcanzar temperaturas termófilas, las que permiten una mejor degradación de la materia orgánica menos lábil y un menor tiempo de secado del material orgánico.

El bioprocesamiento de la mezcla de purines de cerdo con aserrín de pino genera un material sin malos olores, con una carga patógena muy inferior respecto del purín crudo. En el material final obtenido en todos los tratamientos se observa una acidificación, lo cual está en estrecha relación con los altos contenidos de materia orgánica que se presenta al término del periodo de procesamiento, como también con la elevada relación $\mathrm{C} / \mathrm{N}$ que se mantiene en estos materiales, lo cual, sumado a la disminución de la humedad del material estructurante (aserrín), permite suponer que se podría reutilizar con nuevas cargas de purines de cerdo. Los altos niveles de salinidad presente en los purines de cerdo disminuyen notoriamente en el material final y la relación amonio/nitrato, decrece a valores adecuados, que permiten el desarrollo de cultivos sin restricciones.

\section{AGRADECIMIENTOS}

Esta investigación fue financiada con fondos de ASPROCER.

\section{REFERENCIAS}

Boyette, M.D. y otros siete autores; Composting anaerobic swine lagoon sludge and cotton field residue to produce a value-addedproduct. Pp 590-595. In: 2005 Animal Wasted Management Symposium. Research triangle park, North Carolina State University, EEUU. October 5-7 (2005).

Cortina, J., D. Fuentes, A. Valdecantos y G. Casanova; Aplicaciones del compost producido a partir de la fracción sólida del proceso de tratamiento integral de purines SELCO-Ecopurin en la restauración ecológica. Revista científica de porcicultura, Anaporc: (231), 72-90 (2001).

Farres, M.; Gestión de deyecciones ganaderas: minimización en origen y tratamiento. Página circuito porcino (2006). Disponible en: http://mailman.fundacite.arg.gov.ve/pipermail/circuitoporcino-1/ Acceso: 10 de Mayo de 2006.

Faz, A., M. y otros cinco autores; Utilización de purines de cerdo para fertilización de cultivos hortícolas como sustitución de fertilización mineral en Lorca (Murcia). In: I Simposio Nacional sobre control de erosión y degradación del suelo. Madrid, España. Julio 9-11, (2003). Instituto Madrileño de Investigación Agraria. Madrid, España. Disponible en: http://www.wsiam.carm.es/. Acceso: 28 de Abril de 2006

INN; Instituto Nacional de Normalización. Norma Chilena de Compost 2880-2004 (NCh 2880-2004), Compost - Clasificación y requisitos, 23 p. (2004). 
Kirkbride, A.; Anaerobic digested swine manure ideal as corn fertilizer. (2007). Disponible en: http://www. biomassmagazine.com/article-print.jsp?article_id=129. Acceso: 1 Diciembre 2008.

Mathur, P.; Composting processes. In: A.M. Martin (Ed). Bioconversion of waste materials to industrial products. Pag. 147-183. Elsevier Science Publishers, London, England. (1991).

Miralles R., y otros seis autores; Valorización del aporte de estiércol licuado y seco de ganado de porcino en cultivo de estanquillas de olivo. Olivae: 108, 21-30 (2007)

ODEPA; Oficina de Estudios y Políticas Agrarias, Chile. Estadísticas y precios (2006). Disponible en: http://www.odepa.gob.cl. Acceso: 15 de Marzo de 2007.

Peralta, J.M., A. Araya y C. Herrera; Manejo de purines porcinos y tecnologías aplicables: 60-90. In: Peralta, J.M (Ed) INIA - Ministerio de Agricultura. Recomendaciones técnicas para la gestión ambiental en el manejo de purines de la explotación porcina. Colección libros INIA número 18. INIA, Santiago, Chile. 206p. (2005).

Ramírez, G.; Manejo de excretas porcinas-Sistemas convencionales y alternativos. Articulo técnico. (2006). Disponible en: http://www.engormix.com Acceso: 3 de Diciembre de 2006.

TMECC; Test Methods for Examination of Composting and Compost U.S Composting Council Research and education Foundation. (2002). Disponible: http://www.tmecc.org Acceso:30 de Julio de 2006

Smicikles, K. D., P.M. Araya y R.L. Rhykerd; Processed swine manure - Composting and center pivot application to crops. (2008). Disponible en: http://a-c-s-confex.com/crops/2008am/webprogram/ Paper4104.html. Acceso: 1 Diciembre 2008.

Tapia, F.; Estimación de volúmenes de fracción sólida y líquida producidos. Manejo de la fracción sólida. In: Curso plan de aplicación de purines. (2006). Disponible en: http://www.asprocer.cl/ index/publicaciones.asp. Acceso: 10 de Marzo 2007.

Trautmann, T. y E. Olyncin; Cornell Composting Science \& Engineering. (2000). Disponible: http://www.cfe.cornell.edu/compost/microorg.html Acceso: 15 de Abril de 2007.

USEPA - U.S. Enviromental Protection Agency. Standards for the use or disposal of sewage sludge. Fed, Reg. 60:54764-54770. U. S. Gov. Print Office, Washington, D. C. (1995).

Vanotti, M.; Evaluation of Environmentally Superior Technology: Swine Waste Treatment System for Elimination of Lagoons, Reduced Environmentally Impact, and Improved Water Quality. Phase II: Composting of Separated Solids. Final Report. Agriculture Research Service. United States Department of Agriculture (USDA), EEUU. 23p. (2005). Disponible en: http://www.p2pays.org. Acceso: 28 de Abril de 2006.

Varnero, M.T., C. Rojas., R. Orellana y E. Sotomayor; Fitotoxicidad de extractos de residuos orgánicos y su efecto sobre el índice de germinación de rabanito y pepino. Revista Ciencia del Suelo y Nutrición Vegetal: 5(2), 61-66 (2005).

Varnero, M.T., C. Rojas y R. Orellana; Indices de fitotoxicidad de residuos orgánicos durante el compostaje. Revista de la Ciencia del Suelo y Nutrición Vegetal: 7(1), 28-37 (2007).

Zucconi, F. y M. De Bertoldo; Specifications for solid waste compost. Biocycle: May-June, 56-61 (1987).

Zucconi, F., A. Peram, A. Forte y M. De Bertoldi; Evaluating toxicity of inmature compost. Biocycle: 22, 54-57 (1981). 